\title{
Alba Guadalupe Mastache (1942-2004)
}

Robert H. Cobean, Barbara L. Stark et Eric Taladoire

\section{CpenEdition}

Journals

Édition électronique

URL : https://journals.openedition.org/jsa/1778

DOI : 10.4000/jsa. 1778

ISSN : $1957-7842$

Éditeur

Société des américanistes

Édition imprimée

Date de publication : 5 juin 2004

Pagination : 181-188

ISSN : 0037-9174

\section{Référence électronique}

Robert H. Cobean, Barbara L. Stark et Eric Taladoire, «Alba Guadalupe Mastache (1942-2004)»,

Journal de la Société des américanistes [En ligne], 90-2 | 2004, mis en ligne le 17 novembre 2005,

consulté le 04 septembre 2022. URL : http://journals.openedition.org/jsa/1778 ; DOI : https://doi.org/ 10.4000/jsa. 1778 


\section{ALBA GUADALUPE MASTACHE}

(1942-2004)

Le 23 avril 2004 disparaissait brutalement notre collègue, Alba Guadalupe Mastache Flores. Pendant plus de 40 ans, elle a travaillé comme archéologue pour l'Institut national d'anthropologie et d'histoire (INAH) du Mexique. Elle est surtout connue pour ses recherches sur l'urbanisme et l'habitat anciens de la région de Tula (Hidalgo), le cœur de l'État toltèque du Postclassique Ancien (900-1150 A.D.). En réalité, elle s'est toute sa vie passionnée pour son métier et intéressée à de nombreux autres aspects de l'archéologie, de l'histoire ou de l'anthropologie. Son orientation professionnelle était avant tout celle d'une anthropologue qui étayait ses réflexions sur des données archéologiques.

Elle est née à Mérida, Yucatan, le 3 novembre 1942, de parents professeurs dans une école publique qui accueillait de jeunes Mayas yucatèques. Dès son enfance, donc, Guadalupe baigna dans un milieu qui nourrit son intérêt pour les peuples mésoaméricains. Mais elle montra très tôt une attirance comparable pour d'autres civilisations anciennes, avec sa lecture de l'histoire romaine de Mommsen. À l'âge de 15 ans, elle avait lu les œuvres de Plutarque, Tacite et d'autres historiens romains. À 16 ans, elle sortit brillamment de la vénérable Escuela Preparatoria Nacional de Mexico et, la même année, elle s'inscrivit au programme de maestría de l'Escuela Nacional de Antropología e Historia (ENAH). Sa génération fut l'une des dernières de l'âge d'or de l'ENAH. Parmi ses professeurs figurent Wigberto Jiménez Moreno, Pedro Bosch Gimpera, José Luis Lorenzo, Paul Kirchhoff, Barbro Dahlgren, Calixtla Guiteras Holmes, Johanna Faulhaber, Roberto Weitlaner et Francisco de la Maza. Très tôt, elle obtint un premier contrat de recherche comme assistante de Barbro Dahlgren, pour l'analyse des collections ethnographiques d'Asie et de Polynésie (dont beaucoup provenaient d'un don de Miguel Covarrubias) du Musée national d'anthropologie. À 19 ans à peine, Guadalupe obtenait un poste de chercheur permanent à l'INAH. Pour sa maîtrise, elle effectua des travaux de terrain et de laboratoire dans le projet El Infiernillo (Guerrero et Michoacán), sous la direction de José Luis Lorenzo. Sa thèse, Técnicas prehispánicas de tejido, fut publiée par l'INAH, en 1971. Cette synthèse rigoureuse de données archéologiques, ethnohistoriques et ethnographiques, rédigée en 1965-1966, constitue une contribution majeure à la compréhension des techniques préhispaniques du tissage. Pour son étude sur les anciens textiles, Mastache travailla sous la direction experte d'Irmgard Weitlaner Johnson, avec qui elle eut, durant plusieurs décennies, une collaboration fructueuse sur ce thème.

Au début des années 1960, Mastache prit part aux fouilles du projet Teotihuacan, dirigé par Ignacio Bernal. Sa participation révèle bien sa personnalité : 
elle décida de fouiller un secteur que son responsable de chantier considérait comme dénué d'intérêt. Elle découvrit alors le célèbre « Mural des animaux mythologiques » dans l'allée des Morts.

En 1970-1971, Guadalupe Mastache obtint une bourse de l'INAH et du gouvernement italien pour aller étudier au centre de restauration du patrimoine de l'UNESCO, à Rome. Elle en profita pour suivre des cours d'archéologie classique à l'université degli Studi de Rome, ce qui lui permit de participer à des fouilles, à Ostie, l'ancien port de Rome, et dans la chapelle paléochrétienne San Teodoro sur le Forum. Ce séjour changea sa vie : elle se prit d'amour pour tout ce qui était italien et apprit l'italien qu'elle parla couramment. Elle retourna fréquemment en Italie pour de nouvelles visites et y retrouver des amis.

En 1972, Eduardo Matos Moctezuma incita Guadalupe Mastache et Ana María Crespo à s'intéresser à l'occupation préhispanique de la région de Tula (Hidalgo). Cela marqua pour elle le début de plus de trois décennies de recherches sur l'ancienne cité de Tula et l'État Toltèque et d'une longue série de publications. Dès le début de ce programme régional, Mastache collabora avec William T. Sanders et Jeffrey R. Parsons, dont les recherches sur le bassin de Mexico constituèrent le modèle de son étude régionale. La plupart des reconnaissances se déroulèrent heureusement durant les années 1970, un calendrier crucial : dans les années 1980, en effet, des programmes agricoles publics et privés dans la région aboutirent à la destruction de la plupart des sites mineurs. En parallèle, Mastache suivit les cours de doctorat de Sanders à l'université nationale autonome de Mexico (UNAM), et ce dernier fut le directeur de sa thèse, El Estado tolteca : una investigación sobre su proceso de desarrollo y estructura social, económica y politica (1996). Pour sa thèse, Mastache effectua dans les archives des recherches historiques sur les anciennes activités économiques et les systèmes agricoles du Mexique central, qui enrichirent énormément les analyses archéologiques sur Tula. L'Índice de documentos sobre el Centro de México y cartografia antigua sobre el área de Tula, rédigé par Lawrence Feldman et Guadalupe Mastache en 1990, en est une illustration. L'un des résultats significatifs de cette approche pluridisciplinaire fut la découverte que certains des principaux canaux utilisés par les haciendas coloniales de la région avaient été construits sous l'égide de Teotihuacan, entre 200 et 400 apr. J.-C. (Sistemas de riego en el área de Tula, 1976).

À la même époque, Guadalupe effectua un long voyage en Afrique du Nord, visitant le Maroc, l'Algérie et la Tunisie, un séjour inspiré par deux sources très différentes : les cours de William Sanders et les écrits d'Albert Camus. Elle y visita les ruines romaines d'Algérie, décrites de façon fascinante par le romancier. Mais elle étudia aussi les architectures de terre des villes du Maroc que Sanders avait analysées en cours et qui lui fournirent un modèle pour ses recherches ultérieures sur les constructions en adobe de Tula. 
Délaissant provisoirement les Toltèques, Mastache dirigea, en 1979-1980, un projet d'anthropologie sociale pour l'université du Guerrero, qui aboutit à la publication de plusieurs études significatives sur l'artisanat contemporain dans cet État, en particulier l'ouvrage co-signé avec Nora Morett Sánchez (Entre dos mundos: artesanos y artesanías en Guerrero, 1997). Son programme original incluait d'autres aspects comme le folklore, l'activité minière préhispanique et coloniale, ou l'archéologie historique, mais des difficultés administratives avec l'université de l'État l'obligèrent à abandonner. Toutefois, ces années au Guerrero, où était né et avait été élevé son père, Don Eladio Mastache Salgado, furent pour elle comme un retour à la maison.

En 1982, Guadalupe et son mari, Robert Cobean, entamèrent un nouveau projet de l'INAH, Tula and its direct interaction area, destiné à étudier plus en détail l'occupation préhispanique régionale, en utilisant plusieurs méthodes de fouilles et de reconnaissances, des études d'archives historiques et des analyses des systèmes agricoles préhispaniques et coloniaux. Ce projet, étayé par les résultats des travaux antérieurs, aboutit à la publication de cinq volumes, parmi lesquels Tepetitlán : espacio doméstico rural en el área de Tula (1999) et Ancient Tollan : Tula and the Toltec Heartland (2002) représentent le mieux les perspectives de recherche de Guadalupe. Pendant plus de 20 ans, Mastache et Cobean collaborèrent avec Dan M. Healan de l'université de Tulane. Les objectifs personnels de ce dernier pour la fouille de complexes résidentiels et d'ateliers d'obsidienne à Tula se révélèrent cruciaux pour l'approche de la société toltèque. Healan contribua largement aux interprétations et aux analyses quantitatives concernant l'occupation préhispanique de la région de Tula, à des chapitres clés de la thèse de doctorat de Mastache (op. cit.) et au volume publié en 2002 aux presses de l'université du Colorado. Au prix de nombreux mois d'efforts et de collaboration avec Healan et plusieurs géographes, Mastache parvint à transférer sur des cartes numérisées, obtenues depuis des photos satellites, les données d'occupation de la région de Tula (soit plusieurs centaines de sites répartis sur cinq phases). À partir de ces cartes et des riches données de terrain récoltées au fil des reconnaissances, Mastache et ses collègues furent en mesure de publier l'une des meilleures études régionales jamais effectuées en Mésoamérique.

Dans les années 1980, Mastache entama avec succès une nouvelle phase de sa carrière, comme responsable éditoriale de publications archéologiques et de revues. En 1986, en collaboration avec Joaquín García-Barcena, elle fonda la revue Arqueología de l'INAH, dont elle assura la publication pendant 16 ans. Cette revue a rendu accessible au Mexique nombre d'informations anthropologiques fondamentales récentes sur l'Amérique latine, l'Amérique du Nord, l'Asie et l'Europe. Beaucoup d'articles ont été sollicités directement par Mastache, en vue d'enrichir le contenu de la revue et de publier aussi souvent que possible des résultats inédits. En 1993, toujours en collaboration avec Joaquín García- 
Barcena et plusieurs autres collègues, elle fut à l'origine de la revue de divulgation Arqueología mexicana. L'idée originelle remontait à plus de 10 ans, déjà, quand elle en avait discuté avec les responsables de l'INAH et qu'elle avait essayé de les convaincre de la nécessité de rendre accessibles au public les résultats de la recherche archéologique au Mexique. Pendant les premières années d'existence de la revue, elle consacra beaucoup d'énergie à la conception et à l'édition des numéros. Le succès de cette revue, d'excellente qualité scientifique sans pour autant perdre de son accessibilité, témoigne de la justesse des vues de Guadalupe. Cette activité éditoriale se déroula en parallèle avec les responsabilités administratives que notre collègue assumait. Pendant plus de 40 ans, à l'INAH, Mastache occupa diverses fonctions administratives. Dans les années 1960, elle dirigeait le service des permis de fouilles, une fonction qui se rapproche des responsabilités de l'actuel Consejo de Arqueología et du Registro de Zonas y Monumentos Arqueológicos. Plus tard, pendant plusieurs années, elle s'occupa du Departamento de Monumentos Prehispánicos : il s'agissait d'une équipe de plus de soixante archéologues avec laquelle elle mit en place de vastes programmes de fouille et de préservation des 120 sites mexicains ouverts au public. Ce souci rejoint sa volonté de diffusion, de donner à l'opinion une autre image de l'archéologie.

Toujours dans la même optique, à la fin des années 1990, Mastache et Robert D. Drennan (responsable de la SAA Task Force on Latin America) créèrent la série bilingue (espagnol-anglais), publiée conjointement par l'INAH et l'université de Pittsburgh, destinée à rendre disponibles des « reports on primary archaeological research conducted in Mexico, whose results are of importance not only to regional specialists but also to those working on similar subjects in other parts of the world » d'après les propres termes de Drennan and Mastache.

En 1994, elle contribua à l'organisation d'un symposium commun SAAINAH à Mexico, sur la protection du patrimoine culturel dans les Amériques, avec des participants de tout le continent. Par-delà le fléau du pillage, elle était très préoccupée par les nouveaux types de destruction des sites, qui accéléraient leur disparition dans toute l'Amérique latine, en particulier sous l'action des techniques de culture mécanisées. Elle en avait d'ailleurs elle-même été témoin et victime dans sa région d'étude favorite.

L'histoire des recherches archéologiques en Mésoamérique constituait un autre centre d'intérêt pour Guadalupe. Avec Robert Cobean, elle a publié plusieurs essais parmi lesquels " La Arqueología » (in La Antropología en México, vol. 5, 1988), et "Mesoamerican Studies》 (in The Oxford Encyclopaedia of Mesoamerican Cultures, 2001). Dans ses publications, elle accordait toujours une place aux études précédentes, avec le souci de citer tous les précurseurs. Pour Tula, elle avait été impressionnée par la qualité des travaux de Jorge R. Acosta et Hugo Moedano, réalisés il y a plus de 50 ans, et elle travaillait sur une édition de leurs textes, pour une série sur l'archéologie toltèque. 
En 2000-2001, Mastache entama avec William Sanders une série d'échanges et de discussions, relatives aux études comparées des villes préhispaniques, en relation avec les commentaires de ce dernier sur le manuscrit de Ancient Tollan : Tula and the Toltec Heartland. De là naquit l'idée d'un projet original sur l'urbanisme en Mésoamérique, formalisé en 2002, dans le cadre d'un accord entre l'INAH et la Pennsylvania State University. À ce jour, quatre symposia ont réuni plus de 50 spécialistes et un volume bilingue est paru en 2003, sous le titre Urbanism in Mesoamerica (INAH et Pennsylvania State University). Mastache consacra les dernières semaines de sa vie à la préparation des manuscrits du second volume de mémoires des $3^{\mathrm{e}}$ et $4^{\mathrm{e}}$ symposia. Deux autres réunions sont encore prévues, pour la première étape du projet, désormais coordonné par Angel García Cook et William T. Sanders.

Récemment, son intérêt pour l'iconographie et les religions mésoaméricaines s'était accru. Elle avait entrepris des recherches sur les rituels et les probables fonctions politico-religieuses des édifices de l'enceinte monumentale de Tula. Ses perspectives s'étaient élargies, aboutissant à l'hypothèse selon laquelle les principales pyramides de Teotihuacan, Tula et Tenochtitlan étaient probablement dédiées à des divinités similaires, partageant ainsi des fonctions politiques et religieuses spécifiques. Elle y voyait un indice de continuité culturelle sur près de deux millénaires (in "Ancient Tollan : the sacred precinct ", 2000). Dans cette perspective, en 2001, Mastache et Cobean commençaient à Tula un nouveau projet centré sur les fonctions de certains édifices majeurs. Le projet continue malgré son absence.

Recherches, activités éditoriales, responsabilités administratives, souci de rigueur scientifique, mais aussi de rendre l'archéologie plus vivante, plus proche des Mexicains : de quoi remplir une vie, au moins. S'arrêter là pourrait, pour beaucoup, suffire. Ce ne serait pas rendre justice à Guadalupe, que d'oublier l'aspect humain, la chaleur, l'amour de la vie, le sens de l'amitié. On a évoqué plus haut son amour pour l'Italie. On pourrait aussi rappeler sa venue à Leyde pour l'hommage à Ted Leyenaar, l'un de ses vieux amis, ou l'atmosphère chaleureuse qu'elle a su insuffler aux colloques dont elle a assumé la charge. Des heures et des jours de travail, de réflexion, suivis, sans transition, par des conversations amicales, détendues autour de plats traditionnels, témoignage de son profond amour pour son pays.

Outre un projet de fouilles et un colloque, Guadalupe Mastache a laissé en chantier trois livres, deux d'entre eux presque achevés. Ni elle, ni ses proches ne se doutaient de sa maladie. Une convalescence de trois mois lui avait permis de récupérer avec succès d'une opération à l'estomac, quand une nouvelle tumeur apparut : elle est morte à Mexico en avril dernier. Elle laisse ses parents, sept frères et sœurs, treize neveux et nièces et son mari Bob Cobean. L'une de ses nièces est entrée à l'ENAH en 2002. Sa brillante carrière montre comment un chercheur énergique et créatif peut contribuer aussi bien à la recherche scientifique qu'à sa 
gestion, et modifier les relations entre le monde professionnel et le public. Sa famille, ses amis, ses collègues ne peuvent que lui dire « adios » avec tristesse, et continuer sur les traces qu'elle a laissées.

Robert H. COBEAN, Instituto nacional de antropología e historia, Barbara L. STARK, Dept of Anthropology, Arizona State University, et Éric TALADOIRE, Université Paris I

\section{PRINCIPALES PUBLICATIONS}

1971 Técnicas prehispánicas de tejido, INAH, Mexico, coll. « Investigaciones » 20.

1973 «Reconocimiento de superficie en el área de Tula, Hidalgo », Actas de la XIIIa Mesa Redonda de la Sociedad Mexicana de Antropología, Mexico.

1974 « La ocupación en el área de Tula, Hidalgo » (coauteur A. M. Crespo), in E. Matos Moctezuma, éd., Proyecto Tula (primera parte), INAH, Mexico, pp. 71-103, coll. «Científica » 15.

"Textiles from the cueva de la Media Luna, Chiapas, Mexico », Irene Emery Roundtable on Museum Textiles, Proceedings, Archaeological Textiles, The Textiles Museum, Washington D.C.

1975 «Dos fragmentos de tejido decorados con la técnica de plangi », Anales del Instituto nacional de Antropología e Historia, IV, Mexico.

1978 «Sistemas de riego en el área de Tula, Hidalgo », in E. Matos Moctezuma, éd., Proyecto Tula (segunda parte), INAH, Mexico, coll. « Científica » 33.

1981 «La presencia en el área de Tula, Hidalgo, de grupos relacionados con el barrio de Oaxaca en Teotihuacan » (coauteur A. M. Crespo), in E. Rattray, J. Litvak King y C. L. Díaz, éds, Interacción cultural en el México central, UNAM, Mexico.

"La cronología del área de Tula " (coauteurs R. Cobean, A. M. Crespo y C. L. Díaz), in E. Rattray, J. Litvak King y C. L. Diaz, éds, Interacción cultural en el México central, UNAM, Mexico, pp. 187-214.

1982 "El trabajo de la palma en la región de La Montaña, Guerrero » (coauteur N. Morett), Cuadernos de Ciencias Sociales, 4, Universidad Autónoma de Guerrero, Chilpancingo (Guerrero).

Estudios sobre la antigua ciudad de Tula, (coauteurs A. M. Crespo, R. Cobean y D. Healan), Departamento de Monumentos Prehispánicos, INAH, Mexico, coll. "Científica » 121.

«Análisis sobre la traza general de Tula, Hidalgo » (coauteur A. M. Crespo), in Estudios sobre la antigua ciudad de Tula, Departamento de Monumentos Prehispánicos, INAH, Mexico, pp. 11-36, coll. "Científica » 121.

1985 "Tula » (coauteur R. Cobean), in J. Monjarás, R. Brambila y E. Pérez Rocha, éds, El Estado en Mesoamérica, INAH, Mexico, pp. 273-307, coll. «Biblioteca del INAH $»$.

« En defensa del patrimonio cultural : zona arqueológica de Tula », in Primer foro por la defensa del patrimonio cultural, D-11-345, sección 10, Mexico [plusieurs coauteurs]. 
1987 « La cerámica importada en Tula, un informe preliminar », Arqueología, 1, pp. 89132, Departamento de Monumentos Prehispánicos, INAH, Mexico.

1988 « El trabajo de lapidaria en el Estado de Guerrero, una artesanía actual inspirada en formas prehispánicas ", Arqueología, 2, pp. 197-216.

« La Arqueología » (coauteur R. Cobean), in C. G. Mora, éd., La Antropología en México : panorama histórico, INAH, Mexico, vol. V.

« Excavación monumental en Tula » (coauteur R. Cobean), in C. G. Mora, éd., $L a$ Antropología en México : panorama histórico, INAH, Mexico, vol. VI.

1989 "The Coyotlatelco culture and the origins of the Toltec state " (coauteur R. Cobean), in R. A. Dichl et Janet C. Berlo, éds, Mesoamerica after the decline of Teotihuacan : 700-900 A.D., Dumbarton Oaks, Washington D.C.

"The late classic and early postclassic chronology of the Tula region " (coauteur R. Cobean), in D. Healan, éd., Ancient Tula : excavations and survey, University of Iowa Press.

1990 «La cultura Coyotlatelco en el área de Tula » (coauteur R. Cobean), in A. G. Mastache et R. H. Cobean, éds, Las industrias liticas Coyotlatelco en el área de Tula, INAH, Mexico. pp. 7-23, coll. "Científica ".

Índice de documentos sobre el Centro de Mexico y cartografía antigua sobre el área de Tula (coauteur L. Feldman), INAH, Mexico, coll. « Fuentes ».

1991 Tula. Guía oficial de la zona arqueológica (coauteur R. Cobean), INAH-Salvat, Mexico.

1993 «El México antiguo : mundo enigmático y complejo », Arqueología mexicana, 1, pp. 5-13, INAH-Raíces, Mexico.

«El Mexico antiguo : arte, cultura y sociedad » (coauteur R. Cobean), Arqueología mexicana, I (2), pp. 5-9.

1994 «Tula », Arqueologia mexicana, II (7).

1995 Xochicalco y Tula (coauteurs L. López Luján y R. Cobean), CONACULTA, Mexico [édition italienne en 1996, Jaca Book, Milan].

1996 Homenaje a César Sáenz (coauteurs A. García Cook et L. Merino), INAH, Mexico, coll. «Científica ».

"El arte del tejido en el México antiguo », Arqueología mexicana, III (17), pp. 17-25.

"El amaranto y el maguey en la dieta tolteca », Arqueología mesoamericana, I, pp. 355-374, INAH-Raices, Mexico.

"Ancient México » (coauteur R. Cobean), in A. G. Mastache, J. Parsons, R. Santley et C. Serra Puche, éds, Arqueología mesoamericana. Homenaje a William T. Sanders, 2 vols, INAH, Mexico.

1997 Entre dos mundos : artesanos y artesanias en Guerrero (coauteur N. Morett), INAH, Mexico, coll. "Científica ».

« Xochicalco ja Tula » (coauteur R. Cobean), in J. K. Talvitie, éd., Sulka-Kaarme Ja Jaguaari-Jumala, Tampere Art Museum, Tampere, Finland, pp. 210-219.

1999 Tepetitlán : espacio doméstico rural en el área de Tula (coauteurs R. Cobean), Universidad de Pittsburgh-INAH, Mexico, coll. "Bilingue Arqueología de México ».

« Jorge R. Acosta » y « Manuel Gamio » (coauteur R. Cobean), in T. A. Murray, éd., The history of archaeology : an encyclopedia, I, Garland Press, New York. 
« Xochicalco et Tula », Dossiers d'archéologie, 245, pp. 40-51, Dijon.

" Un taller de alfareros en la antigua ciudad de Tula » (coauteurs C. Hernández, R. Cobean et M. E. Suárez), Arqueología, segunda época, 22, pp. 69-88, INAH, Mexico.

2000 "Coyotlatelco, Toltec culture y Tula region » (coauteur R. Cobean), in S. T. Evans et D. Webster, éds, The archaeology of ancient México : an encyclopedia, Garland Press, New York.

"Ancient Tollan : the sacred precinct » (coauteur R. Cobean), RES, 38, pp. 101133, Harvard University.

2001 "Archaeology in México » (coauteur R. Cobean), in T. A. Murray, éd., The history of archaeology : an encyclopedia, II, Garland Press, New York.

" Mesoamerican studies, Toltec y Tula » (coauteur R. Cobean), in D. Carrasco, éd., The Oxford encyclopaedia of Mesoamerican cultures, Oxford University Press, New York.

2002 Ancient Tollan. Tula and the Toltec Heartland (coauteurs R. Cobean et D. M. Healan), University Press of Colorado, Boulder.

Sous presse

Las ofrendas del Palacio Quemado : turquesa y concha en un palacio Tolteca (coauteur R. Cobean), University of New Mexico Press, Albuquerque.

El mundo Tolteca. Interacción interregional durante el Postclásico Temprano (coauteur R. Cobean), University of New Mexico Press, Albuquerque. 